EPJ Web of Conferences 62, 05003 (2013)

DOI: $10.1051 /$ epjconf/20136205003

(C) Owned by the authors, published by EDP Sciences, 2013

\title{
The VERDI fission fragment spectrometer
}

\author{
M.O. Frégeau ${ }^{1}$, T. Bryś ${ }^{1}$, Th. Gamboni ${ }^{1}$, W. Geerts ${ }^{1}$, S. Oberstedt ${ }^{1, a}$, A. Oberstedt ${ }^{2}$, and R. Borcea ${ }^{3}$ \\ ${ }^{1}$ European Commission, Joint Research Centre, Institute for Reference Materials and Measurements \\ (IRMM), 2440 Geel, Belgium \\ ${ }^{2}$ Fundamental Fysik, Chalmers Tekniska Högskola, 41296 Göteborg, Sweden \\ ${ }^{3}$ Depart. Nucl. Phys., IFIN-HH, 77127, Bucharest-Magurele, Romania
}

\begin{abstract}
The VERDI time-of-flight spectrometer is dedicated to measurements of fission product yields and of prompt neutron emission data. Pre-neutron fission-fragment masses will be determined by the double time-of-flight (TOF) technique. For this purpose an excellent time resolution is required. The time of flight of the fragments will be measured by electrostatic mirrors located near the target and the time signal coming from silicon detectors located at $50 \mathrm{~cm}$ on both sides of the target. This configuration, where the stop detector will provide us simultaneously with the kinetic energy of the fragment and timing information, significantly limits energy straggling in comparison to legacy experimental setup where a thin foil was usually used as a stop detector. In order to improve timing resolution, neutron transmutation doped silicon will be used. The high resistivity homogeneity of this material should significantly improve resolution in comparison to standard silicon detectors. Post-neutron fission fragment masses are obtained form the timeof-flight and the energy signal in the silicon detector. As an intermediary step a diamond detector will also be used as start detector located very close to the target. Previous tests have shown that poly-crystalline chemical vapour deposition (pCVD) diamonds provides a coincidence time resolution of $150 \mathrm{ps}$ not allowing complete separation between very low-energy fission fragments, alpha particles and noise. New results from using artificial single-crystal diamonds (sCVD) show similar time resolution as from $\mathrm{pCVD}$ diamonds but also sufficiently good energy resolution.
\end{abstract}

\section{Prompt neutron multiplicities and mass distributions}

The last years have seen the development of many nuclear applications, such as nuclear reactors of $4^{\text {th }}$ generation, spallation source etc. requiring precise nuclear data for reactions induced by neutrons with energy going up to $20 \mathrm{MeV}$. Pre-neutron mass and total kinetic energy (TKE) of fragments produced in neutron-induced fission are routinely measured at the European Commission Joint Research Center (EC JRC-IRMM) by means of twin Frisch-grid ionization chambers, where the energy of both fragments is measured. Thanks to the momentum conservation it is possible to infer the pre-neutron mass, but it is necessary to correct for prompt neutron emission. To do this correction, it is assumed that fragments are

\footnotetext{
ae-mail: stephan.oberstedt@ec.europa.eu
}

This is an Open Access article distributed under the terms of the Creative Commons Attribution License 2.0, which permits unrestricted use, distribution, and reproduction in any medium, provided the original work is properly cited. 
at an equal temperature. The number of neutrons emitted can then be inferred based on existing data. However, it was suggested that in some case the equal temperature approximation does not hold: for ${ }^{237} \mathrm{~Np}[1]$ and ${ }^{235} \mathrm{U}$ [2] most of available energy coming from the incident neutron will lead to enhanced neutron emission from the heavy fragment. It was also shown that, following the chosen hypothesis variations in the fission yield could reach up to $30 \%$ for very asymmetric mass division [3]. Considering the important consequences that these corrections have on the mass yields it is very important to do systematic measurements on many systems and at different excitation energies to better understand prompt neutron emission from fission fragments.

Another motivation to measure accurately mass and energy distributions of fission fragments emerges from the need to more efficiently model the fission process for evaluation of nuclear data. Many models that can predict neutron multiplicities for different systems are in development but for the moments they all require experimental fission-fragment mass and kinetic energy data as input parameter [4-6]. Unfortunately, for the moment, input data with sufficient accuracy are scarce and the excitation energy range is limited. With the help of these models and good input data it will be possible to provide more accurate evaluated data where experimental data are missing.

\section{Verdi}

Fission fragment mass can be determined by the measure of its time-of-flight (TOF) and its kinetic energy. TOF is given by the time required for the fragment to travel between a start detector and a stop detector. Kinetic energy is measured directly by standard detector such as ionization chamber or silicon detector. Mass is then calculated with relevant kinematic equations. The obtained mass corresponds to the fragment mass after neutron emission, also called post-neutron mass. It is possible to evaluate the fragment mass before the emission of neutron or pre-neutron mass. The TOF of both fragments should then be measured whilst knowing the mass of the fissioning system. Once more the momentum and energy conservation is used but no correction is required to obtain the pre-neutron and post-neutron masses. This technique is often referred to as the $2 \mathrm{E}-2 \mathrm{~V}$ method. The neutron multiplicity is then simply given by the difference between the pre-neutron and post-neutron masses.

The reference for a $2 \mathrm{E}-2 \mathrm{~V}$ experimental setup is COSI-FAN-TUTE [7] with a resolving power of $\mathrm{m} / \delta \mathrm{m}=170$ for $\mathrm{m}=95$. The time of flight of the fission fragments was measured between two thin carbon foils where micro channel plates were detecting the secondary electrons. The energy was then measured by an ionization chamber. Unfortunately, its limited geometrical acceptance resulted in very low coincidence efficiency, especially when fission fragments lost co-linearity due to neutron emissions. Also one of the main limitations to the energy and mass resolution came from energy straggling induced by the thin carbon foils used for the TOF measurement and by the entrance window of the ionization chamber.

VERDI (VElocity foR Direct particle Identification) aim at achieving comparable performance as COSI-FAN-TUTE but with a geometric efficiency of twice $1 \%$. Simple simulations indicate that in the actual configuration, with a flight path of $50 \mathrm{~cm}$ length and an energy resolution of $0.5 \%$, a $90 \mathrm{ps}$ timing resolution is required to obtain a mass resolution $\mathrm{m} / \delta \mathrm{m}>125$. VERDI will determine the arrival time of the fragment with the time information provided by the silicon detector used for the energy measurement. Any need for energy-loss corrections is therefore strongly reduced. However, to obtain very good timing resolution with a large area silicon detector is very challenging. We are going to use neutron transmutation doped (NTD) silicon for the stop detectors. The very high resistance homogeneity of this material should allow us to significantly improve the timing properties of the $450 \mathrm{~mm}^{2}$ detector that will be used. Similar detectors, using regular silicon, have already been used in conjunction with a poly crystalline start detector, and they allowed us to achieve a mass resolution of 3-4u.

Silicon detectors cannot be used as start detector: they are too thick to allow passage to fragments and neutron dose received close to the target would damage them very rapidly. Instead two different 
Fission 2013

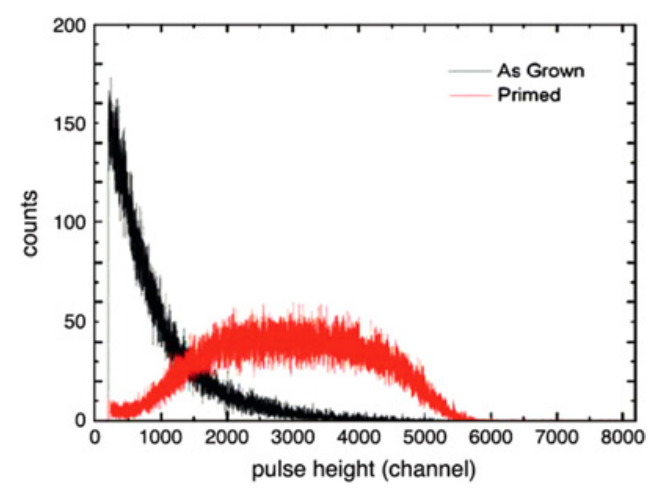

Figure 1. Pulse height spectrum from a triple alpha source detected by a $1 \times 1 \mathrm{~cm}^{2}$ poly-crystalline diamond (thickness: $100 \mu \mathrm{m}$ ) in as grown state (black) and in primed state (red) [13].

start detectors will be used. The first one is a $300 \mu \mathrm{m}$ thick single crystal diamond detector located very close to the target, on the side opposite to the flight path. It will be used as the start detector in a $2 \mathrm{E}-\mathrm{V}$ configuration, i.e. that the time of flight is measured for only one of the fragments. In this configuration only the post-neutron mass of fission fragment can be determined directly. The independent $2 \mathrm{E}$ measurement is equivalent to that with an ionization chamber. The combination of both data allows crosschecking the applied neutron correction. The start detector has to show excellent timing properties, high neutron hardness and also relatively good energy resolution. Extensive investigation of diamond detectors is currently being performed at IRMM and will be the subject of the $3^{\text {rd }}$ section of this article. Since it is not possible yet to make very thin diamond detector that would allow fragment to cross them we have to use a different detector for the $2 \mathrm{E}-2 \mathrm{~V}$ configuration. This second detector will be composed of an electrostatic mirror and a micro-channel plate used to detect secondary electrons emitted by the target when fission fragments are emitted. This is not considered in the following.

\section{Diamond detectors}

\subsection{Poly-crystalline diamond detector}

Diamond is one of the materials with the highest charge mobility, despite their intrinsic energy resolution significantly lower than for silicon (conversion factor of $12.86 \mathrm{eV} / \mathrm{e}-\mathrm{h}$ [8] measured for scCVD diamonds) their timing properties are excellent: rise time being shorter than one nanosecond. Diamonds also show an excellent resistance to radiations, $14 \mathrm{MeV}$ neutron doses of up to $8 \times 10^{14}$ neutron/ $\mathrm{cm}^{2}$ can be absorbed before significant damage is observed [9]. Several pCVD diamonds were used in a first series of tests. First were $100 \mu \mathrm{m}$ thick and had an active surface of $1 \times 1 \mathrm{~cm}^{2}$, second had thickness of $180 \mu \mathrm{m}$. The latter had an anode segmented in 4 strips in order to reduce the capacitance of the detector and therefore improve its timing capabilities. When used in their as grown state pCVD diamonds show very bad charge collection efficiency due to the presence of many defects and traps acting as recombination centers. In Figure 1 the black curve show the energy response of the diamond for alphas particles coming from a mixed nuclide ${ }^{239} \mathrm{Pu},{ }^{241} \mathrm{Am},{ }^{244} \mathrm{Cm} \alpha$-source. The pulse height spectrum show important variations and most of the pulses have less than $20 \%$ of the maximum pulse height. With such an energy response it would not be possible to differentiate between an alpha particle, a fission fragment or even background. Fortunately it is possible to fill the traps responsible for electron-hole recombination by irradiating the diamond with electrons.

The curve in red shows the same energy response after irradiations for few hours with a strong ${ }^{90} \mathrm{Sr} /{ }^{90} \mathrm{Y}$ beta source. It was then possible to separate the alphas from the background even though 


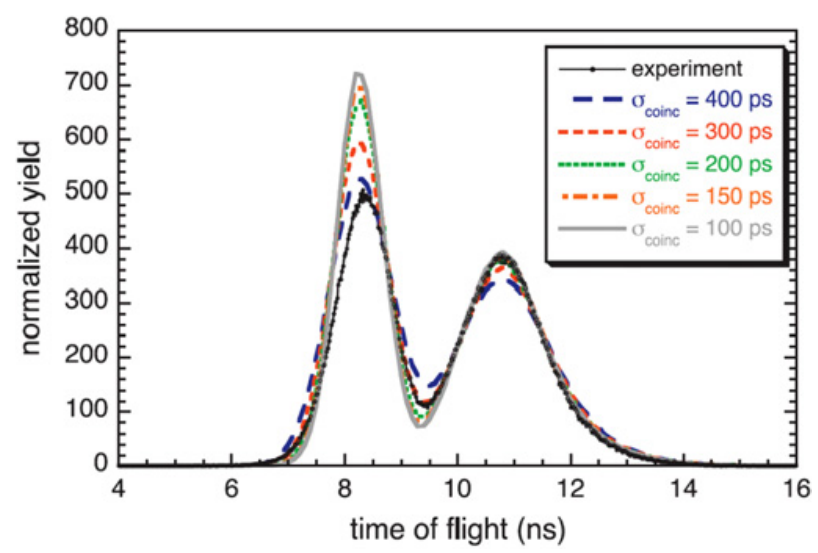

Figure 2. Time of flight spectrum of fission fragments from the spontaneous fission of ${ }^{252} \mathrm{Cf}$ measured by two 4-fold segmented poly-crystalline diamond detectors (thickness: $180 \mu \mathrm{m}$ ) on a flight path of $11.2 \mathrm{~cm} \mathrm{[13].}$

energy resolution is not sufficient to separate the three alpha energies. It is to note that this primed state is stable for many days, as long as the diamond is not exposed to UV light.

To test the intrinsic time resolution we used a simple setup where 2 similar pCVD diamond detectors are place facing one to each other, separated by a distance of $11.2 \mathrm{~cm}$. On a first one a californium source is mounted at about $2 \mathrm{~mm}$ from the diamond so the difference in fragments TOF detected by this detector is as small as possible. The ${ }^{252} \mathrm{Cf}$ was evaporated on a $250 \mathrm{~nm}$ thick Ni backing so the fission fragments can be detected by both detectors, fragments going to the closest detector had to cross the backing. Figure 2 shows the corresponding TOF spectrum. Fission fragments show a rather broad mass and energy distribution making thus the time resolution impossible to measure directly. In order to infer the intrinsic time resolution of the detector from the measured TOF spectrum we used a Monte Carlo simulation using masses and energies from 2E measurements [10-12]. TOF broadening was then introduced for different arbitrary time resolution, shown by the different colored curves in Figure 2. The simulation better reproducing the TOF spectrum was found to be the one with $150 \mathrm{ps}$ coincidence timing resolution corresponding to an intrinsic time resolution better than $110 \mathrm{ps}$. Despite the very good reproduction of the spectrum part corresponding to the heavy fragments (the longest TOF) it was not possible for the simulation to reproduce the part of the spectrum corresponding to the lighter fragments (the shortest TOF). This discrepancy was explained by the fact that the fragments with low kinetic energy hitting the detector close to the fission source, deposits an energy, which lies below the trigger threshold of the LED module and is, therefore, not detected. It was observed that this phenomenon was more affecting light fragments then heavy fragments. For complete discussion of these data please report to [13].

\subsection{Single-crystal diamond detector}

To overcome limitation coming from energy response of pCVD diamond we are currently testing detectors made of single-crystal chemical vapor deposition (sCVD) diamonds. These crystals are almost traps free and charge collection distance ranging up to $300 \mu \mathrm{m}$ is routinely obtained. To our knowledge the biggest SCVD detector-grade diamonds measure $4.6 \times 4.6 \mathrm{~mm}^{2}$. To make a detector of similar size to the poly-crystalline one we used 4 diamonds place one next to the others in a square configuration. Figure 3 shows a typical pulse obtained from an alpha particle collected with a broadband current sensitive pre-amplifier. It shows a very fast rise time shorter than $1 \mathrm{~ns}$ and a pulse with of about $4 \mathrm{~ns}$. 


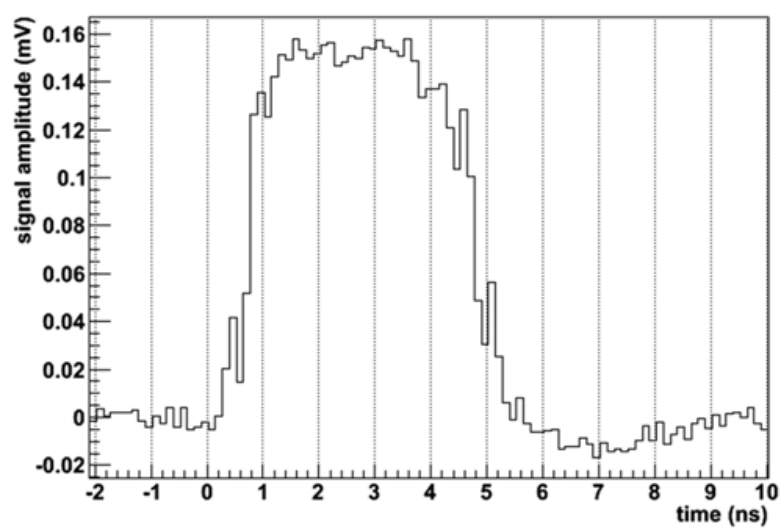

Figure 3. Typical pulse shape for an alpha particle in a single-crystal diamond, where the charge was collected by a broadband current sensitive pre-amplifier and digitized at a sampling speed of 8 Gs.

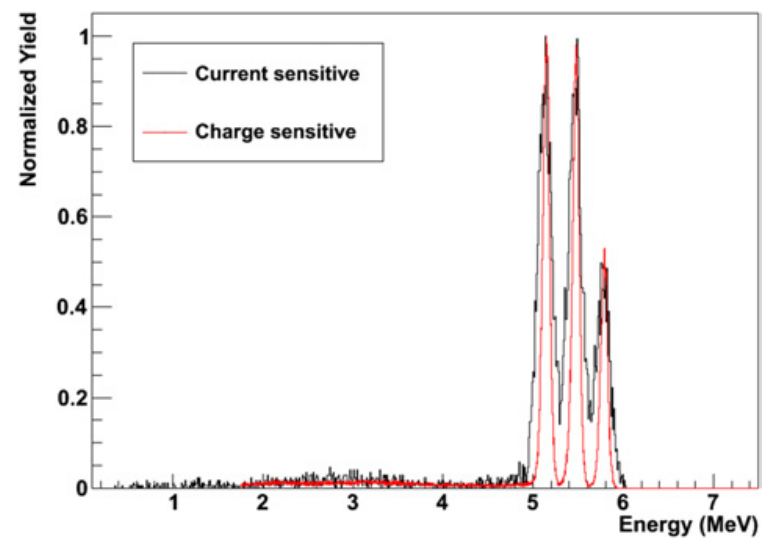

Figure 4. Pulse height spectrum for a 3alpha source and a sCVD diamond detector. Charges collected with charge sensitive pre-amplifier (red) and current sensitive pre-amplifier (black).

The relatively flat top of the pulse indicated that very little charge recombination occur during electronsholes drift in the diamond.

Energy resolution of sCVD diamond detector was tested with a mixed nuclide $\alpha$-source. The corresponding energy spectrum is depicted in Figure 4. With the help of a charge sensitive pre-amplifier (red curve) it was possible to measure a pulse width FWHM of $129 \mathrm{keV}$ at $5.1 \mathrm{MeV}$. We also explored the possibility to use broadband pre-amplifiers (PA) and digitizers to benefit from the good timing properties that should come from the broadband PA, and at the same time measure the energy of the particle. The energy determined this way, shown in black, has clearly not as good resolution as the one taken with the charge sensitive PA, i.e. around $180 \mathrm{keV}$ FWHM, but would be sufficient to discriminate between noise, $\alpha$ particle and fission fragment.

The intrinsic time resolution of the SCVD diamond detectors was measured with a similar setup as used for pCVD diamond detectors but over a flight path of $8.78 \mathrm{~cm}$. A first TOF spectrum is shown in Figure 5 (black) together with a Monte Carlo simulation (red) similar to the one used for pCVD diamond with the same time resolution of $150 \mathrm{ps}$. It can be observed that, as expected, the relative height of the two peaks is similar to the one measured with the $2 \mathrm{E}$ methods. 


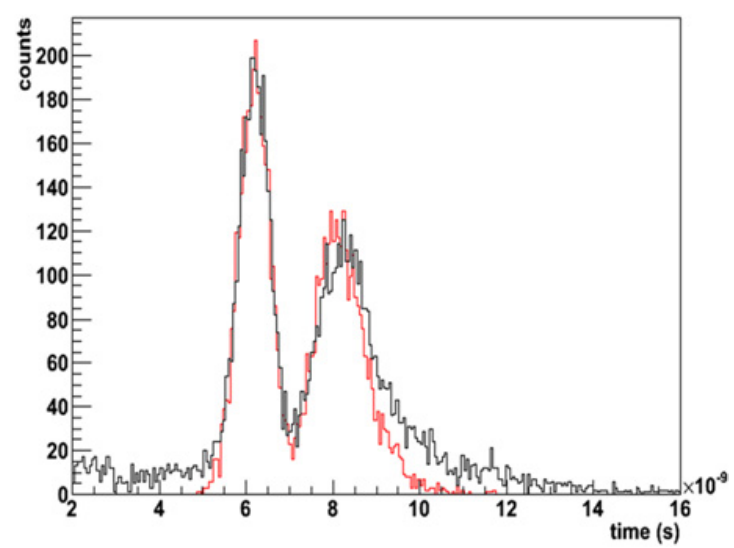

Figure 5. Time of flight spectrum of fission fragments from the spontaneous fission of ${ }^{252} \mathrm{Cf}$ measured by singlecrystal CVD diamond detectors over a flight path of $8.78 \mathrm{~cm}$ (black) and Monte Carlo Simulation based on a coincidence time resolution of $150 \mathrm{ps}$ (red).

\section{Conclusions}

During the development and construction of the TOF spectrometer VERDI sCVD diamond detectors show to be very promising for detection of fission fragments, achieving similar timing resolution as pCVD diamond detector but with much better energy resolution. Their use solved the problem of detection efficiency observed for lighter fragment with pCVD. In the future, we intent to determine what are the time resolution that can be obtained with a full digital acquisition system. Tests with timing optimized NTD silicon will also begin soon.

\section{References}

[1] A. Naqvi, F. Käppeler, F. Dickmann, and R. Müller, Phys. Rev. C. 34, 218 (1986)

[2] R. Müller, A. A. Naqvi, F. Käppeler, and F. Dickmann, Phys. Rev. C. 29, 885 (1984)

[3] A. Al-Adili, F.-J. Hambsch, S. Pomp, and S. Oberstedt, Phys. Rev. C. 86, 054601 (2012)

[4] A. Tudora, Ann. Nucl. Ener, 53, 507 (2012)

[5] B. Becker, P. Talou, T. Kawano, Y. Danon, and I. Stetcu, Phys. Rev. C. 87, 014617(2013)

[6] J.P. Lestone, Nuclear Data Sheets 112, 3120 (2011)

[7] A. Oed, P. Geltenbort, R. Brissot, F. Gönnenwein, P. Perrin, E. Aker, and D. Engelhardt, Nucl. Inst. Meth., 219, 569 (1984)

[8] M. Pomorski et al., phys. stat. sol. (a) 202, 2199 (2005)

[9] M. Angelone et al., Rev. Sci. Inst. 77, 023505 (2006)

[10] E. Birgersson et al., Nuclear Physics A 791, 1 (2007)

[11] F.-J. Hambsch, S. Oberstedt, Nuclear Physics A 617, 347 (1997)

[12] F.-J. Hambsch, private communication, 2004

[13] S. Oberstedt et al., Nucl. Inst. and Meth. In Phys. Res. A 714, 31 (2013) 